\title{
Injection Effect of Anti-CD3 Monoclonal Antibody on Primo Vessel in Lymph Vessel of Rabbit with Lipopolysaccharide-Induced Inflammation
}

\author{
Sang-Heon Choi, Jong-Gu Choi, Sang-Suk Lee* \\ Department of Oriental Biomedical Engineering, College of Health and Medical Sciences, Sangji University, Wonju, Korea
}

Received September 10, 2021

Revised December 6, 2021

Accepted January 17, 2022

\section{Correspondence to}

Sang-Suk Lee

Department of Oriental Biomedical Engineering, College of Health and Medical Sciences, Sangji University, Wonju, Korea

E-mail sslee@sangji.ac.kr
Background: The primo vascular system can be viewed as a circulatory system that plays a therapeutic function in regenerating the body tissue. The anti-CD3 monoclonal antibody was used as an immunotherapeutic agent to treat the novel coronavirus infection (COVID-19).

Objectives: In this study, we observed the effect of injecting lymph nodes with Foralumab, an anti- human CD3 epsilon therapeutic monoclonal antibody, on primo vessels.

Methods: The structure and atomic stoichiometry of the antibody were determined by transmission electron microscopy and energy dispersive spectroscopy. Alcian blue dying solution was injected into the lymph nodes of the abdominal vena cava of rabbits, and the solution further flowed into the lymph vessels.

Results: A primo vessel with primo nodes stained with Alcian blue was clearly visible in the lymph vessel. By injecting Foralumab into lymph nodes of rabbits with lipopolysaccharide-induced inflammation, the floating primo vessel in the lymph vessel appeared thicker and was distinctly visible.

Conclusion: The observation of the primo vessel post-treated with Foralumab in the inflamed lymphatic system suggests the possibility of a functional role of the primo vascular circulatory system in pathophysiological conditions.

Keywords: Primo vessel, Lipopolysaccharide (LPS), Anti-CD3, Monoclonal antibody, Foralumab, Lymph vessel

\section{INTRODUCTION}

In the past two decades, the primo vascular system (PVS) has been represented by research studies as a meridian circulatory system [1-3]. In response to the demand of individuals among the aging population who wish to live a healthy life, convergence research on PVS and the meridian circulatory system has been steadily established along with advances on these topics in the field of biotechnology $[4,5]$. Investigation of the anatomical significance of the meridian circulatory system began in 1965 , with the discovery of the Bong-Han vessel circulatory system by a research team led by North Korean scientist Dr. Kim Bong-Han [6,7]. Then, in the early 2000s, the name "primo circulatory system" was re-examined by a research team led by Professor KwangSup Soh of Seoul National University [1,4]. Recently, studies on the development of genetic markers and antibodies using nanotechnology application in the separation and purification of primo vessels in lymph vessels, metabolism of primo vessels, and functions of primo vessels in the brain and skin were published by many research teams worldwide [1-5].

The vascular circulatory system carries nutrients and oxygen to cells in living things, and the lymphatic circulatory system functions in eliminating pathogens and viruses. However, the PVS can be viewed as a circulatory system that plays a therapeutic function in regenerating the body tissues [8]. The current strategies for achieving cytokine inhibition include cytokine receptor inhibition and depletion of specific immune cells in addition to steroid therapy [9]. Monoclonal antibody therapy is one of the methods used to deplete specific immune cells. It suppresses $\mathrm{T}$ cells through antibodymediated complement activation and removal of immune conjugates, and can be used to specifically deplete IFN- $\gamma$ secreting $T$ cells. However, this therapy is administered as an intravenous injection of an anti-CD3 monoclonal antibody, and since it targets all $\mathrm{T}$ cells, it induces significant 
immunosuppression and has side effects such as high fever and chills $[10,11]$.

In this study, anti-CD3 monoclonal antibody, Foralumab [12], was used as an immunotherapeutic agent to treat the novel coronavirus infection (COVID-19) [10]. The antibody was injected into the lymphatic system of rabbits, in which inflammation was induced using lipopolysaccharide (LPS) [13], to observe the morphological characteristics of primo vessels in the lymph vessels. Furthermore, as a conjugated construct of Foralumab-based nanoparticles, Foralumab is necessary for effective immunotherapy in order to suppress the overactivation of $\mathrm{T}$ cells. The preparation of the antibody to be administered involved the use of an optimized liquid phase that induces a suitable stimulus to the target along with an appropriate route of administration and dosage. This study aimed to observe the morphological characteristics of primo vessels seen as the injection effect of Foralumab in rabbits with lipopolysaccharide-induced inflammation.

\section{MATERIALS AND METHODS}

\section{Sample preparation}

The rabbit dissection experiment in this study was conducted according to the regulations of Nano-Bio laboratory (No. 2021-02) of Sangji University, and the protocols used in this study were reviewed and approved by the Animal Ethics Committee (IACUC) of Sangji University, Wonju-city, Republic of Korea. Ten-week-old New Zealand female rabbits, weighing $1.8 \mathrm{~kg}$, supplied by Daehan Biolink Co., Ltd. (Eumseong, Chungcheongnam-do, Republic of Korea) were used in the study [14]. To obtain the primo vessel from the lymph vessel of the rabbit, the following steps were conducted and recorded [15]: Step 1: An anesthetic prepared by mixing zoletil $(1.5 \mathrm{ml})$ with rompun $(0.5 \mathrm{ml})$ was injected into the leg muscles of the rabbit. Step 2: The hair on the abdomen of the anesthetized rabbit was removed using an epilator along the midline of the abdomen from the bladder to the stomach. Step 3: LPS, Alcian blue (AB), and monoclonal antibody $(\mathrm{mAb})$ were injected into the lymph node near the abdominal vena cava in the relevant order according to the given amount. A $0.3 \mathrm{ml}$ syringe was used to dispense $90 \mu \mathrm{L}$ of Alcian blue, and $0.5 \mathrm{ml}$ of LPS at $200 \mathrm{~g} / \mathrm{kg}$ concentration was injected into the lymph node of the abdominal vena cava. Step 4: The dye was injected along the lymph node, and was expected to spread to the lymph vessels and travel along with the lymph fluid, which would turn the dark-blue dye into a pale color. Only the thin primo vessels in the lymph vessels were stained blue. Then, the video recording was saved.

\section{Preparation of the anti-CD3 monoclonal antibody}

The anti-CD3 human monoclonal antibody 'Foralumab' used in this study has the antibody structure of the antihuman CD3 epsilon therapeutic antibody-endotoxin $<1 \mathrm{EU} /$ mg manufactured by Creative Biolab [16]. Foralumab (0.1 $\mathrm{mg} / \mathrm{ml}$ in physiological saline phosphate-buffered saline, PBS) was administered at once into the abdominal vena cava lymph node of an inflamed rabbit over 20 min after $\mathrm{AB}$ administration.

\section{Analysis and observation of the antibody and primo vessel}

As determined by the transmission electron microscopy (TEM) image analysis of the junction structure of the antiCD3 monoclonal antibody, the binding elements were investigated by energy dispersive spectroscopy (EDS). After preparing a humanized antibody from the anti-CD3 monoclonal antibody, a humanized mouse monoclonal antibody, it was administered to rabbits to maintain the same affinity level to allow animal experiment performance. Images of the PVS under a stereomicroscope (SZX12; Olympus, Tokyo, Japan) were captured using a chargecoupled device camera (DP70; Olympus, Tokyo, Japan). For all other experiments, dissection was performed by the previously described process of anesthesia [17].

\section{RESULTS AND DISCUSSION}

A cytokine storm is a phenomenon that occurs when proinflammatory cytokines are over-secreted and is considered dangerous because when it caused, our immune cells to not only kill viruses and virus-infected cells, but also attack healthy cells in our body. $\mathrm{T}$ cells play a key role in causing this cytokine storm.

When an antigen-presenting cell (APC) and T cell come into contact, two signals must be sent to activate the T cell. The first signal is sent when MHC II of APC binds to the T cell receptor (TCR), and the second is sent when APC B7 binds to T cell CD28. As shown in Fig. 1A, T cells are activated when these two signals are combined; this triggers the secretion of pro-inflammatory cytokines $[12,18]$. Foralumab as anti-CD3 monoclonal antibody (Fig. $1 \mathrm{~B}$ and $1 \mathrm{C}$ ) functions by binding to the $\mathrm{CD} 3$ receptor in the TCR complex $[19,20]$. Our body has several regulatory systems that work to prevent the immune response from overreacting. When $\mathrm{T}$ cells are activated, pro-inflammatory cytokines such as interleukin (IL)-2, IL-2R, and IR-4 as well as anti-inflammatory cytokines such as IL-10 and IR-4 are simultaneously secreted. These anti-inflammatory cytokines also suppress the immune response of immune cells such as 
macrophages [10].

In addition, there is a limit to the number of $\mathrm{T}$ cells activated. The cytotoxic T lymphocyte antigen-4 (CTLA4), an immune checkpoint inhibitor, binds to B7 in APC, preventing it from binding to the CD28 receptor. This process is significant since $\mathrm{T}$ cells can be activated only when $\mathrm{B} 7$ of APC and CD28 of T cells bind to together. Most CTLA-4 is present in T cells, but when MHC and TCR bind together, depending on the intensity of the stimulation sent to $\mathrm{T}$ cells, CTLA-4 either rises or does not rise to the surface of T cells. The weaker the binding signal, the lower the number of CTLA-4 on the surface of T cells and the higher the number of activated T cells. When the MHC and TCR binding signal is stronger, a higher number of CTLA-4 rises to the surface of $\mathrm{T}$ cells, inhibiting $\mathrm{T}$ cell activation. Therefore, among $\mathrm{T}$ cells, regulatory $\mathrm{T}$ cells continuously attach CTLA- 4 to the surfaces of cells and suppress T cell activation [18].

The actual shape and TEM image of Foralumab are shown in Fig. 2, respectively. Foralumab functions by binding to CD3 in the T cell complex and inhibiting the cytokine storm in three major ways. The first is by antigenic modulation.
When the antibody binds to the receptor, the receptor is removed from the cell surface and eliminated, preventing the activation of $\mathrm{T}$ cells. The receptor either falls off by shedding or is internalized and absorbed. In the second method, if Foralumab binds to an already activated $\mathrm{T}$ cell, the cell achieves a state wherein it does not react even when an antigen is injected into a living body, a phenomenon called anergy. CTLA4 is released to halt the activation of the T cell through incapacitation of the cell. Third, Foralumab causes apoptosis and also secretes TGF- $\beta$, a cytokine that kills T cells. Therefore, $\mathrm{T}$ cells secrete the cytokine FoxP3 to block FoxP3 differentiation, and macrophages clean up the already oxidized $\mathrm{T}$ cells and block the differentiation of effector $\mathrm{T}$ cells to create tolerogenic dendritic cells. FoxP3 begins to suppress effector $\mathrm{T}$ cells by creating tolerant dendritic cells [16].

A field emission TEM (JEM-F200, JEOL Ltd, Japan) was used to observe the bonding characteristics between Foralumab and magnetic nanoparticles. Appropriate amounts of the sample were loaded into the TEM grid, and the TEM images obtained showed that the antibody included $\mathrm{C}, \mathrm{N}, \mathrm{O}$,
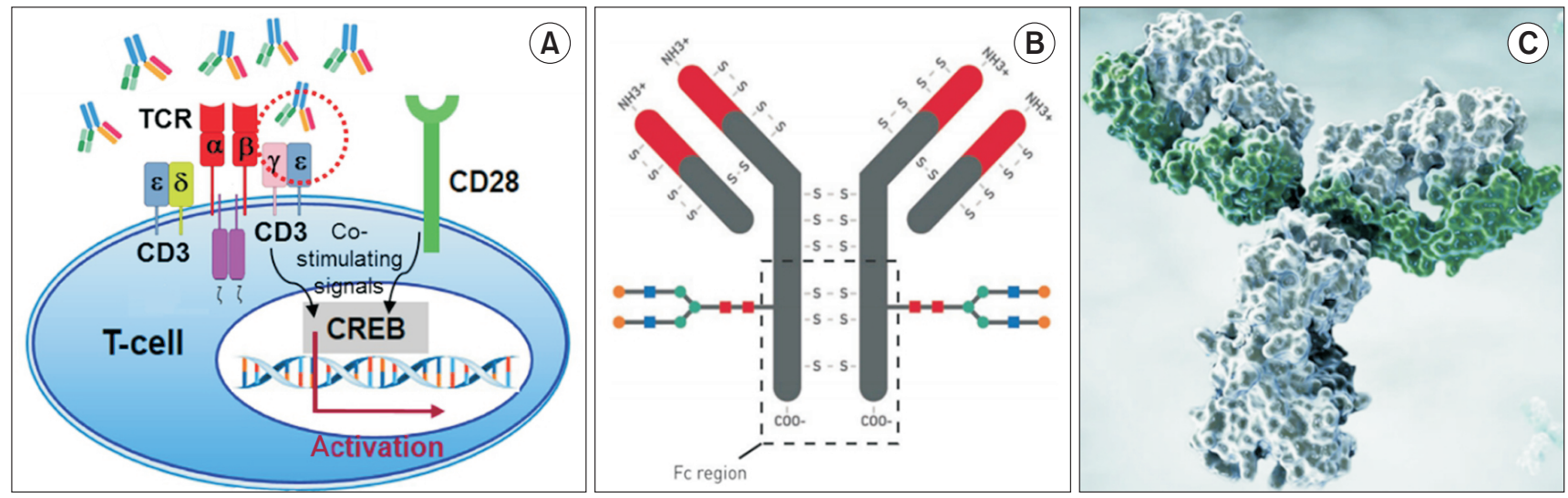

Fig. 1. (A) Schematic diagram of cytokine-secreting T-cell activation by binding antibodies to CD3 and CD28 [18]. (B) Hypothetical structure of a 100\% humanized anti-CD monoclonal antibody (Foralumab) [19]. (C) Schematic diagram of the Fc-directed antibody and constituent elements of the antibody [20].
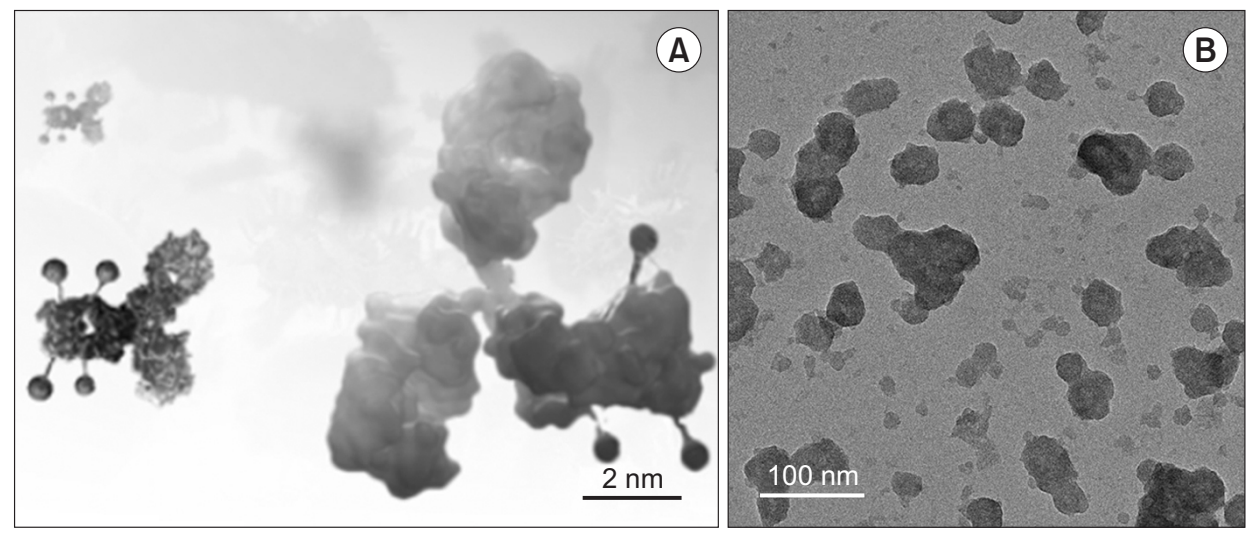

Fig. 2. (A) Actual shape and (B) tunneling electron microscope image of Foralumab, a 100\% humanized anti-CD3 monoclonal antibody. 
A
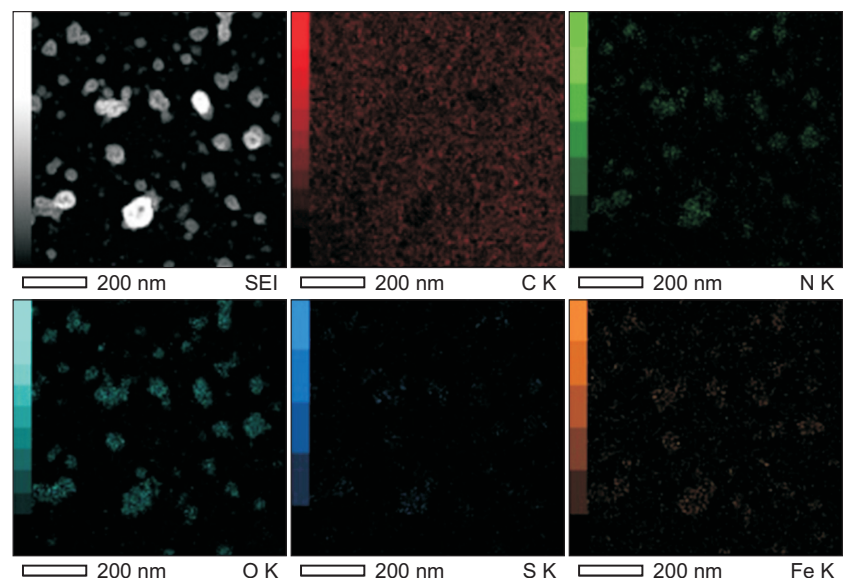

B

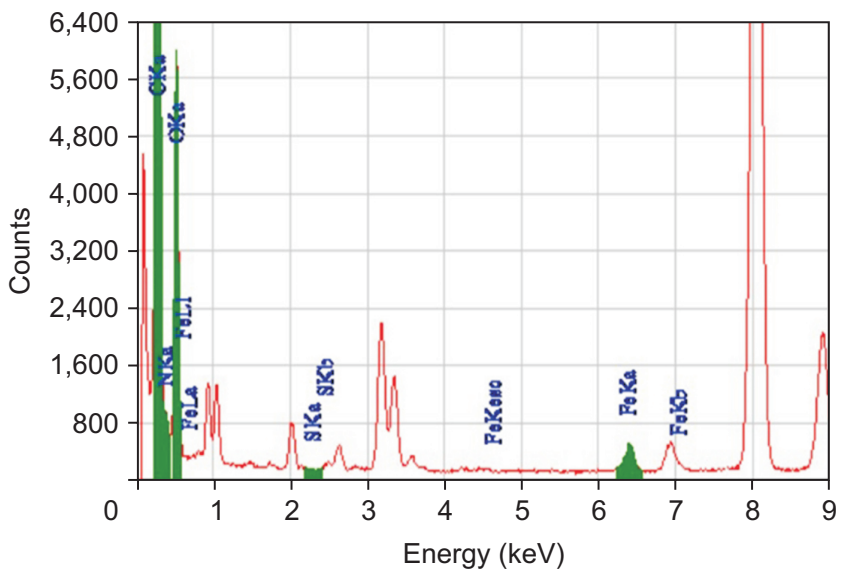

Fig. 3. (A) Transmission electron microscopy (TEM) image and atomic intensities of $\mathrm{O}, \mathrm{S}$, and Fe for Ab. (B) The energy dispersive spectroscopy spectrum for Foralumab from TEM and atomic stoichiometric analyses. The $S$ element is a major component constituting the frame of the Foralumab antibody. The characteristic energy for the S element is $2.307 \mathrm{keV}$.
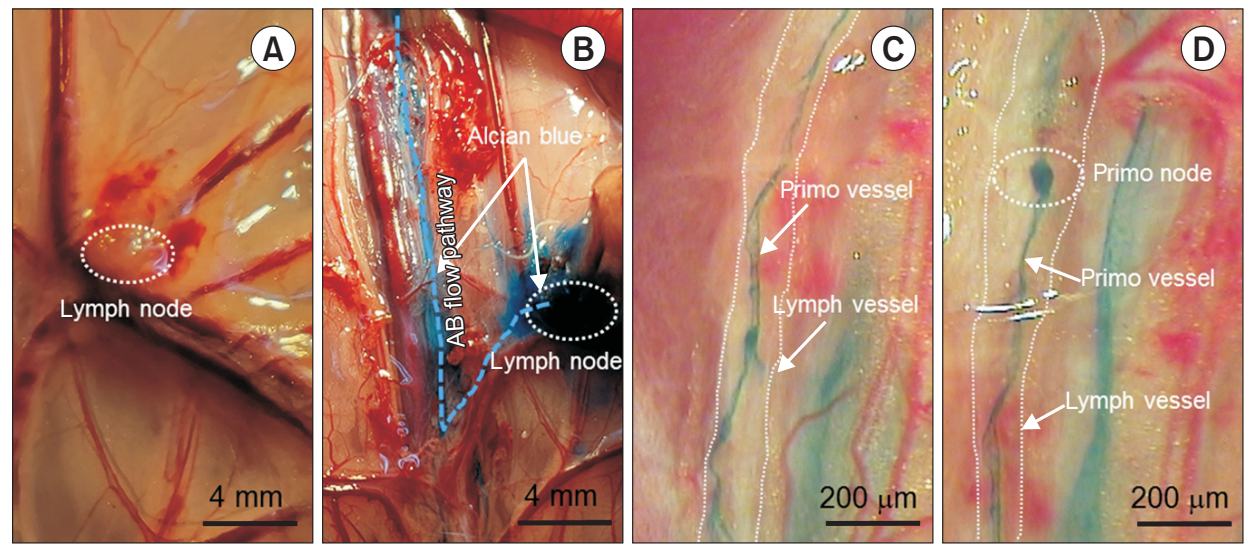

Fig. 4. Lymph node in vena cava of a rabbit (A) before and (B) after Alcian blue $(\mathrm{AB})$ staining. (C) Floating primo vessel and (D) primo node inside a lymph vessel. White lines and circles indicate lymph and primo nodes, and arrows indicate lymph and primo vessels, respectively.

and S elements. The TEM images for Foralumab present in the samples were subjected to rapid EDS with high sensitivity. The intensity images for the main elements $\mathrm{C}, \mathrm{N}, \mathrm{O}, \mathrm{S}$, and Fe are shown in Fig. 3A. As per the EDS spectrum shown in Fig. $3 \mathrm{~B}$, the characteristic energy of the $\mathrm{S}$ element contained in the major frame of Foralumab is $2.307 \mathrm{keV}$.

Primo vessel observed in the lymph vessel through dissection experiments in rabbits is shown in Fig. 4A, and the dissection was performed around the lymph nodes and lymph vessels of the abdominal vena cava. It was injected into the lymph node of the abdominal vena cava as shown in Fig. 4B and delayed for about $10 \mathrm{~min}$ before flowing into the lymph vessel. Fig. 4 shows the presence of the primo vessel in the lymph vessel, which was observed as Alcian blue only stained the primo vessel. The node of the primo vessel is shown in Fig. 4D, and it is seen as a clear corpuscle. The diameters of the lymph and primo vessels shown here were observed to be $100 \mu \mathrm{m}$ and $25 \mu \mathrm{m}$, respectively. The shape and structure of the primo vessel in the lymph vessel have been shown in previous studies conducted by this research team $[14,15]$.

Primo vessel observation before and after treatment using therapeutic anti-CD3 monoclonal antibody for doseinduced rabbit injection of LPS was performed. Before the observation based on their response to the LPS injection [13], the morphological and structural characteristics of the primo vessels, such as their thickness and lengths, connected to the suspected primo node were obtained. Some representative photographs of typically long primo vessels are shown in Fig. $4 \mathrm{C}$ and $4 \mathrm{D}$. In rabbits, several primo vessels were observed an AB solution, which flowed into the lymph node and slowly exited through an abdominal lymph vessel. The lengths and diameters of the lymph vessels ranged from $5-7 \mathrm{~cm}$ and 250 $300 \mu \mathrm{m}$, respectively. The primo vessel's structure could be seen because the dye had attached itself to the wall of the primo vessel and did not interfere with the flow of the lymph fluid. And the lengths and diameters of the primo vessels ranged from $2-3 \mathrm{~cm}$ and $25-30 \mu \mathrm{m}$, respectively. 

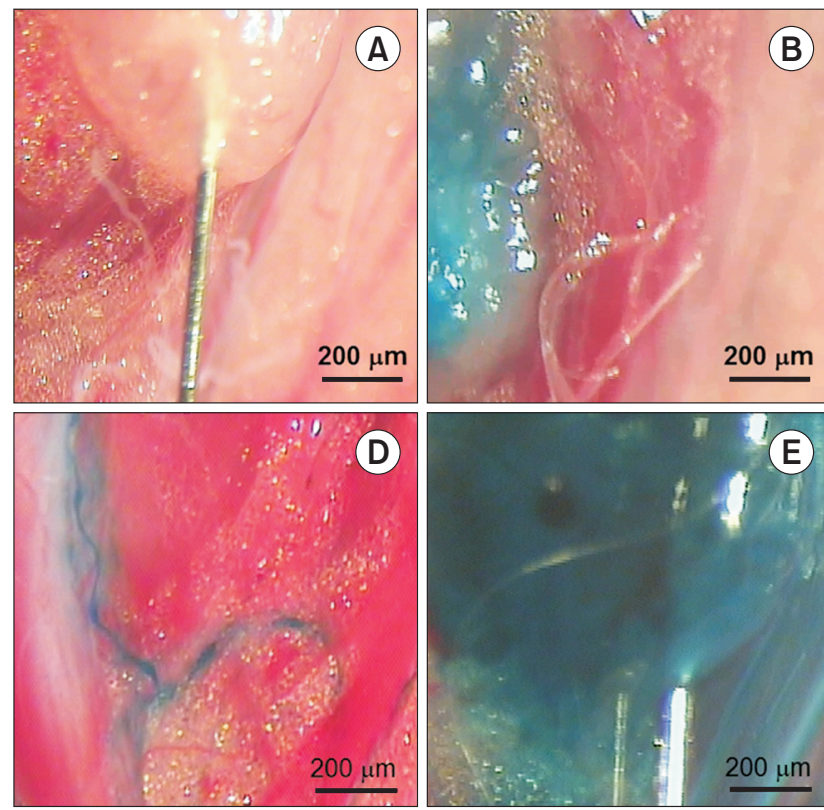

Inflammation was induced by injecting LPS into the rabbit lymph node, and changes in the primo vessels were observed. Fig. 5A the injection of LPS into the lymph node of the abdominal vena cava [15]. Fig. 5B and 5C show an enlarged view of the injection of $90 \mu \mathrm{L} A B$ into the same lymph node immediately after LPS injection, as shown in Fig. 4B. After injection of the antibodies, it was confirmed that the bubbles slowly spread to the lymph node. Foralumab in PBS was administered to the abdominal vena cava lymph node of an inflamed rabbit, and changes in the primo vessel were observed (Fig. 5D). Fig. 5D shows a primo vessel in which only LPS has been administered before Foralumab administration, while Fig. 5E shows the administration of Foralumab into the lymph node of the abdominal vena cava.

Fig. 5F shows the primo vessel imaged at the same location as that shown in Fig. 5D, 10 min after LPS and Foralumab administration. As shown in Fig. 5F, the primo vessel was much thicker, darker, and more clearly visible, likely due to the activation of $\mathrm{T}$ cells in the lymphatic system of inflamed rabbits induced by the antibody treatment. Whether the morphological changes in the primo vessel induced by the injection of LPS and Foralumab, which conjugates with T-cells, result from antibody-induced immune activity needs to be investigated through further experiments.

\section{CONCLUSIONS}

Foralumab, used as an immunotherapeutic for COVID-19, works by binding to CD3 in the T-cell complex, which inhibits a cytokine storm through three major processes: antigenic modulation, anergy, and apoptosis. Our study showed that injecting the Foralumab into the lymph node

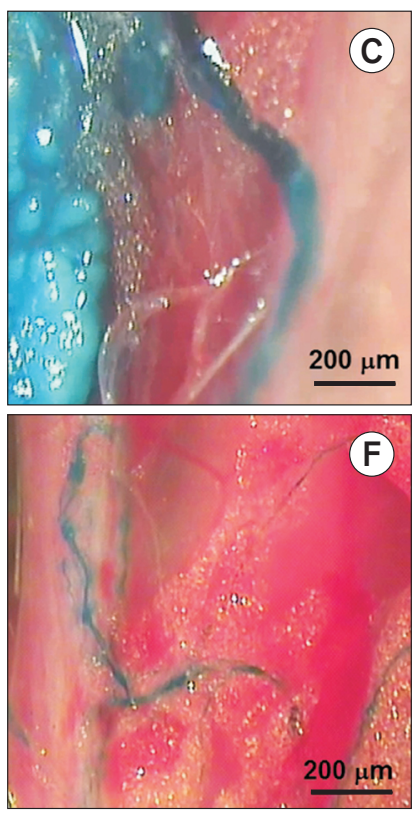

Fig. 5. (A) Image of lipopolysaccharide (LPS) injection into the abdominal vena cava lymph node of a rabbit, (B) image of Alcian blue $(A B)$ injection into lymph node, $(C)$ image of $A B$ spreading into the lymph vessel after its injection into the lymph node, (D) image of primo vessel in the lymph vessel after injection of LPS and AB into the lymph node, (E) image of Foralumab antibody injection into the lymph node, and (F) image of the primo vessel after treatment with Foralumab antibody in LPSinduced rabbit. of rabbits inflamed with LPS resulted in a thicker and an apparent floating primo vessel, indicating that Foralumab induced changes in the lymphatic immune system. Our findings suggested a possible functional role of the PVS in pathophysiological conditions.

\section{FUNDING}

This research was supported by Basic Science Research Program through the National Research Foundation of Korea (NRF) funded by the Ministry of Education (2021 R1I1A3054773).

\section{AUTHORS' CONTRIBUTIONS}

Conceptualization: Lee SS; Data curation: Choi SH, Lee SS; Formal analysis: Choi SH, Lee SS; Funding acquisition: Lee SS; Investigation: Choi SH, Choi JG; Methodology: Choi SH, Choi JG; Project administration: Lee SS; Resources: Lee SS; Supervision/Validation: SS LEE; Writing (Original/review/ editing): Lee SS.

\section{CONFLICT OF INTEREST}

The authors declare no conflict of interest.

\section{ORCID}

Sang-Heon Choi, https://orcid.org/0000-0002-4706-180X Jong-Gu Choi, https://orcid.org/0000-0002-2347-3228

Sang-Suk Lee, https://orcid.org/0000-0002-4706-180X 


\section{REFERENCES}

1. Soh KS. Bonghan circulatory system as an extension of acupuncture meridians. J Acupunct Meridian Stud 2009;2:93-106.

2. Lim CJ, Shen Y, Choi MC, Ryu PD. Primo bundles identified by microcomputed tomography in primo vascular tissue on the surface of rat abdominal organs. J Acupunct Meridian Stud 2020;13:136-45.

3. Vodyanoy V, Pustovyy O, Globa L, Sorokulova I. Primovascular system as presented by Bong Han Kim. Evid Based Complement Alternat Med 2015;2015:361974.

4. Soh KS, Kang KA, Harrison DK. The Primo Vascular System: Its Role in Cancer and Regeneration. New York: Springer, 2011.

5. Kwon BS, Ha CM, Yu S, Lee BC, Ro JY, Hwang S. Microscopic nodes and ducts inside lymphatics and on the surface of internal organs are rich in granulocytes and secretory granules. Cytokine 2012;60:587-92.

6. Kim BH. [The Kyungrak system]. J Jo Sun Med 1965;108:1-38 Korean.

7. Kim BH. Kyungrak system and theory of sanal. Bristol: Curious Pages Publishin, 2019.

8. Lim CJ, Shen Y, Lee SY, Ryu PD. Potential erythropoiesis in the primo-vascular system in heart failure. Adv Exp Med Biol 2017; 977:409-15

9. Stefanov M, Potroz M, Kim J, Lim J, Cha R, Nam MH. The primo vascular system as a new anatomical system. J Acupunct Meridian Stud 2013;6:331-8.

10. Ledford H. Antibody therapies could be a bridge to a coronavirus vaccine - but will the world benefit?. Available at: https:// www.nature.com/articles/d41586-020-02360-y [Date accessed: August 11, 2020]

11. Lee JS, Park S, Jeong HW, Ahn JY, Choi SJ, Lee H, et al. Im- munophenotyping of COVID-19 and influenza highlights the role of type I interferons in development of severe COVID-19. Sci Immunol 2020;5:eabd1554.

12. Ogura M, Deng S, Preston-Hurlburt P, Ogura H, Shailubhai K, Kuhn C, et al. Oral treatment with foralumab, a fully human anti-CD3 monoclonal antibody, prevents skin xenograft rejection in humanized mice. Clin Immunol 2017;183:240-6.

13. Yücel G, Zhao Z, El-Battrawy I, Lan H, Lang S, Li X, et al. Lipopolysaccharides induced inflammatory responses and electrophysiological dysfunctions in human-induced pluripotent stem cell derived cardiomyocytes. Sci Rep 2017;7:2935.

14. Noh YI, Rho M, Yoo YM, Jung SJ, Lee SS. Isolation and morphological features of primo vessels in rabbit lymph vessels. J Acupunct Meridian Stud 2012;5:201-5.

15. Lee HR, Rho MS, Hong YJ, Ha YE, Kim JY, Noh YI, et al. Primo vessel stressed by lipopolysaccharide in rabbits. J Acupunct Meridian Stud 2015;8:301-6.

16. Creative BioLabs. Foralumab overview. Available at: https:// www.creativebiolabs.net/foralumab-overview.htm [Date accessed: September 20, 2021]

17. Park DY, Lee HR, Rho MS, Lee SS. Effective isolation of primo vessels in lymph using sound- and ultrasonic-wave stimulation. J Acupunct Meridian Stud 2014;7:298-305.

18. Humancells Biosciences. Human Cord Blood CD3+ Pan T Cells. Available at: https://humancellsbio.com/products/humancord-blood-cd3-pan-t-cells [Date accessed: September 25, 2021]

19. Thermo Fisher Scientific. SiteClick ${ }^{\mathrm{TM}}$ Antibody Labeling Kits. Available at: https://www.thermofisher.com/order/catalog/ product/S10455\#/S10455 [Date accessed: October 15, 2021]

20. Tiziana Life Sciences. Foralumab. Available at: http://www. tizianalifesciences.com/drug-pipeline/foralumab/ [Date accessed: October 24, 2021] 